\title{
Evaluación de la trombolisis dirigida por catéter para el manejo de la trombosis venosa profunda iliofemoral extensa
}

\author{
Evaluation of catheter-directed thrombolysis for iliofemoral deep venous extensive \\ thrombosis
}

\author{
- Andrés Acevedoํ, Hugo Páez², Aníbal Morillo³, Alberto Muñoz', Guillermo Quintero', Myriam Rodríguez' \\ Andrés Felipe Cardona 5,6 \\ 'Grupo Hematología y Trasplante de Médula Ósea, Instituto de Oncología, Fundación Santa Fe de Bogotá (Bogotá, Colombia); investigador asociado Hemo-ONCOLGroup. \\ ¿División de Educación, Fundación Santa Fe de Bogotá (Bogotá, Colombia). \\ Dénósticas, Fundación Santa Fe de Bogotá (Bogotá, Colombia).

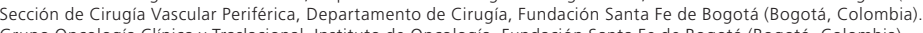

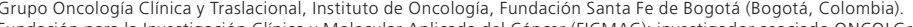

\section{Resumen}

Introducción: La trombosis venosa profunda iliofemoral extensa (TVPIF) tiene un importante impacto a largo plazo en términos de insuficiencia venosa crónica e incapacidad secundaria. La trombolisis dirigida por catéter (TDC) puede reducir la morbilidad asociada a este evento.

Materiales y métodos: Se diseñó y proyectó un registro monográfico de seguimiento, que incluyó una serie de pacientes tratados con TDC en la Fundación Santa Fe de Bogotá (FSFB) entre el 2008 y 2011. Se describieron las características clínicas de los pacientes, el procedimiento y los desenlaces a corto y a largo plazo.

Resultados: La TDC se realizó en cuatro pacientes con TVPIF izquierda, tres mujeres y un hombre; la mediana de edad fue 58 años (rango 35 a 70). En tres casos, se documentó un síndrome de May-Thurner asociado (SMT). Las trombosis agudas y subagudas, caracterizadas por ultrasonografía, se encontraron cada una en dos pacientes. La TDC se llevó a cabo utilizando alteplase y tenecteplase en uno y tres pacientes, respectivamente. Al finalizar la TDC, todos los casos catalogados con SMT fueron manejados con angioplastia por balón más stent (ABS). El éxito trombolítico fue completo en los dos casos de TVPIF aguda, y parcial en los dos casos de TVPIF subaguda. Solo hubo un evento hemorrágico mayor y la permeabilidad del vaso a largo plazo se mantuvo en los cuatro pacientes, sin eventos trombóticos reincidentes o reestenosis. No se diagnosticó síndrome postrombótico en ninguno después de seis meses de seguimiento.

Conclusiones: La TDC para la TVPIF es un procedimiento factible de efectuar en nuestra institución, ofreciendo una frecuencia aceptable de complicaciones con importantes beneficios clínicos a largo plazo. En los casos de TVPIF y SMT, el uso concomitante de ABS pudo mantener la permeabilidad venosa durante el tiempo de seguimiento.

Palabras clave: terapia trombolítica, trombosis venosa, síndrome de May-Thurner, cateterismo periférico.

\begin{abstract}
Introduction: lliofemoral extensive deep venous thrombosis (IFDVT) has an important clinical long-term impact when considering chronic venous insufficiency and related disability. Catheter-directed thrombolysis (CDT) might reduce IFDVT associated morbidity. Materials and methods: We performed a monographic follow-up registry of CDT treated patients at Fundación Santa Fe de Bogotá (FSFB) between 2008 and 2011. We described clinical characteristics, procedure aspects and short plus long-term outcomes. Results: CDT was performed in four patients for left IFDVT, three women and one man, with a median age of 58 years (range, 35-70). Three presented May-Thurner syndrome (MTS). Acute and subacute thromboses were characterized by ultrasonography each one in two patients. CDT was executed using alteplase and tenecteplase in one and three patients, respectively. All MTS cases were additionally managed with ballon angioplasty plus stenting (BAS), immediately after CDT. Complete thrombolytic success was evident in two acute cases and partial was evident in the two subacute cases. There was only one mayor but successfully controlled bleeding event in an elderly patient. Long-term venous patency by Doppler ultrasonography, without recurrent stenosis or thrombosis, was conserved in all. No postthrombotic syndrome was diagnosed after a minimum 6-month follow-up period. Conclusions: CDT for IFDVT is a valid option to be performed at FSFB, offering acceptable complication frequency, but most importantly, relevant long-term clinical benefits. In MTS associated IFDVT cases, venous patency was maintained by concomitant use of BAS during the whole follow-up.
\end{abstract}

Key words: thrombolytic therapy, venous thrombosis, May-Thurner syndrome, peripheral catheterization. 


\section{Introducción}

La trombosis venosa profunda (TVP), parte del espectro del tromboembolismo venoso (TEV), se define como la oclusión total o parcial del flujo sanguíneo en una vena profunda por la formación de un coágulo intraluminal'. Tanto por su frecuencia como por sus consecuencias, la TVP es una condición médica relevante; de acuerdo con un estudio nacional en múltiples centros hospitalarios de los Estados Unidos entre el 2007 y 2009, por cuenta de esta condición se realizaron cerca de 350.000 hospitalizaciones cada año en la población adulta, determinando una tasa anual de 152 ingresos hospitalarios por cada 100.000 habitantes $^{2}$. Lo anterior se traduce en una incidencia anual de TVP entre 1.0 y 1.6 casos por cada 1.000 habitantes $^{3}$. En general, la trombosis suele ocurrir con mayor frecuencia en las extremidades inferiores, aunque también se puede encontrar en los miembros superiores, en la circulación esplácnica y en la cerebral ${ }^{4}$. Según su localización, la oclusión del sistema venoso de las extremidades ocurre en el $80 \%$ de las TVP de los casos sintomáticos en las venas poplíteas y en aquellas más proximales ${ }^{5}$.

Fisiológicamente, la trombolisis endógena es un proceso secuencial. Una vez ocurrida la TVP y por medio de los activadores del plasminógeno, se enciende el sistema fibrinolítico, que permite una regresión eficiente del trombo alojado en la circulación proximal hacia la segunda semana después del evento. Progresivamente, hacia la semana 24 y 36 resta cerca de un cuarto de la carga trombótica inicial. Durante todo este tiempo, el sistema es regulado por los inhibidores de los activadores del plasminógeno. Una vez finalizado el proceso, estos se encargan de restaurar el balance hemostático inicial. A pesar de su eficiencia, cuando ocurre en la circulación venosa proximal de los miembros inferiores, la fibrinólisis endógena logra la resolución completa del trombo únicamente en cerca de la mitad de los pacientes, dejando una carga trombótica residual sobre las venas ${ }^{6}$.

Adicionalmente, las trombosis extensas que se alojan en el eje iliofemoropoplíteo se han ligado a una menor probabilidad de aclaramiento y recanalización del segmento afectado 7 , junto a una menor probabilidad de normalizar el retorno venoso y su función valvular, medidos por ecografía Doppler después de un año ${ }^{8}$. Varios estudios observacionales han identificado una clara relación entre la carga trombótica residual luego de tratar el evento agudo y su correspondiente impacto a largo plazo, incluyendo la probabilidad de mantener la permeabilidad del vaso, la funcionalidad venosa (reflujo sanguíneo y obstrucción), la complejidad y la gravedad de la sintomatología, la incapacidad resultante (calidad de vida) y los costos asociados al tratamiento a largo plazo $^{9-14}$

Este espectro clínico y fisiopatológico fundamentado en la insuficiencia venosa crónica (IVC) se ha denominado como síndrome postrombótico (SPT) y se caracteriza por un amplio conjunto de síntomas y signos manifestados por diversos grados de dolor, edema, pigmentación de la piel o, incluso, ulceración de la extremidad ${ }^{15}$. Hasta la mitad de los pacientes que han sufrido TVP pueden desarrollar este síndrome en algún punto de su evolución, implicando una importante repercusión clínica ${ }^{15}$. La fisiopatología del SPT está ligada a tres fenómenos concurrentes: la hipertensión venosa persistente, producto del reflujo sanguíneo; la insuficiencia de las válvulas venosas y la obstrucción persistente del lumen venoso por el trombo (figura 1$)^{16}$.

La cronificación de estos fenómenos desencadena procesos inflamatorios que resultan en la remodelación estructural del vaso y en la modificación de diversas propiedades de sus paredes (la fibrosis, la pérdida de la capacitancia venosa y el flujo de salida), lo que finalmente repercute en la funcionalidad ${ }^{17,18}$.

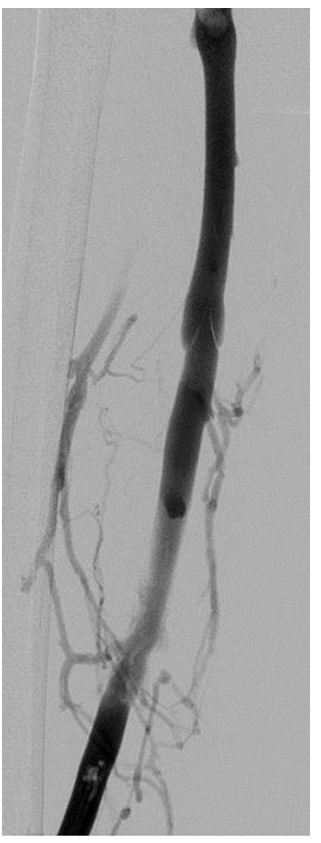

Se observa el sistema valvular sobre el segmento ilioemoral venoso permeable con circulación colateral en un paciente con trombosis venosa profunda y síndrome de May-Thurner. Todo el conjunto permite el paso exitoso del medio de contraste hacia la vena cava inferior La enfermedad tromboembólica persistente afecta y remodela la estructura del vaso, generando la insuficiencia venosa crónica, que se manifiesta clínicamente como el síndrome postrombótico.

Figura 1. Venografía del segmento iliofemoral izquierdo. 
Más aún, el efecto crónico se presenta con mayor frecuencia y gravedad en quienes han tenido trombosis que comprometen las venas iliacas o femorales ${ }^{19}$. A pesar de tratarse con anticoagulación plena, entre el $50 \%$ y $80 \%$ de los pacientes con TVP iliofemoral desarrollan algún grado de IVC20,21, para lo cual el uso diario de medias de compresión graduada (MCG) logra reducir la incidencia de SPT en una cuarta parte de los sujetos ${ }^{22-24}$. Con una población afecta predominantemente productiva (media de edad de 56 años) o mayor, resulta indispensable la prevención de las secuelas del SPT a largo plazo ${ }^{25}$.

El tratamiento de la TVP tiene en cuenta varios objetivos. A corto plazo, se requiere detener la extensión del trombo, prevenir que partes de este se desprendan y se embolicen a otros lechos vasculares, y, por lo tanto, prevenir las muertes asociadas al TEV. A largo plazo, es necesario reducir el riesgo de recurrencia de los TEV y de reestenosis, preservar la función valvular, repermeabilizar completamente la obstrucción cuando hay persistencia del trombo y prevenir el desarrollo de la IVC. Como consecuencia, la terapia estándar comprende la anticoagulación mediante la heparinización sistémica durante el episodio agudo, seguida de anticoagulación oral con warfarina ${ }^{26}$. Además, está claramente justificado el uso concomitante de las $\mathrm{MCG}^{26}$.

Desde hace casi 60 años, se propuso la eliminación temprana del agente vascular lesivo por diversos métodos para evitar la remodelación, el daño valvular y la obstrucción crónica, lo que se ha denominado como terapia trombolítica ${ }^{27}$. El principal objetivo de esta consiste en remover la carga tromboembólica, acelerando su degradación, para restaurar la permeabilidad veno$\mathrm{sa}^{27}$. Más recientemente, los avances de la radiología intervencionista y en medicina vascular han permitido el desarrollo de estrategias mecánicas, farmacológicas o mixtas para cumplir dicho objetivo. Las primeras comprenden la extracción quirúrgica del coágulo (trombectomía), que, en una proporción importante, no logran retirar el trombo en su totalidad. Además, se relacionan con una alta incidencia de reestenosis y morbilidad secundaria ${ }^{28}$. Sin embargo, la trombectomía aún resulta de utilidad como último recurso, cuando no es posible efectuar otras estrategias trombolíticas y la carga trombótica es relevante.

Por otro lado, en la actualidad se han desarrollado dispositivos endovasculares capaces de alcanzar el trombo para removerlo físicamente del lumen de la vena. Cambiando de principio, las alternativas farmacológicas implican la infusión de agentes capaces de acelerar enzimáticamente el proceso de disolución del coágulo ${ }^{27}$. Las diferentes modalidades de trombolisis farmacológica comprenden, según la distribución del agente, la infusión sistémica, regional o local del trombolítico; esta última, también denominada trombolisis dirigida por catéter (TDC), consiste en una intervención guiada por imágenes en la que un agente trombolítico es dispuesto directamente sobre el trombo a través de un catéter y/o un alambre colocado dentro de la vena ocluida, donde se concentra el agente sobre el coágulo ${ }^{29}$. Propuestas recientes comprenden el uso concomitante de diversos mecanismos físicos y químicos aplicados en distintos momentos del procedimiento, buscando mejorar la eficacia de la trombolisis.

A continuación, se describe la experiencia obtenida tras la realización de TDC en cuatro pacientes con trombosis venosa profunda iliofemoral (TVPIF) extensa localizada en algún miembro inferior, en la Fundación Santa Fe de Bogotá (FSFB).

\section{Materiales y métodos}

Se diseñó un registro monográfico de seguimiento para incluir a todos los pacientes llevados a TDC por TVPIF en la FSFB, a partir de enero del 2008 hasta diciembre del 2011. La información se registró utilizando un formato único para tal fin, el cual incluyó una serie de variables consensuadas entre los departamentos de imágenes diagnósticas, hematología y cirugía vascular periférica. Dos de los investigadores (AA y HP) registraron y analizaron los datos empleando el paquete estadístico SPSS versión 18.0 (IBM ${ }^{\circledR}$ software, Chicago, IL, EE. UU.). Los resultados se caracterizaron mediante la determinación de frecuencias absolutas, relativas, medidas de tendencia central y de dispersión. Se consideraron diversas variables relacionadas con la clínica (edad, sexo, síntomas y signos de TVP, características ecográficas y extensión del trombo), los procedimientos técnicos asociados con la TDC (tiempo y dosis del trombolítico, terapias concomitantes), los resultados a corto plazo reportados en menos de 30 días (éxito trombolítico, complicaciones embólicas y hemorrágicas, mortalidad durante la TDC) y a largo plazo informados después de seis meses (recurrencias 
trombóticas, reestenosis, permeabilidad venosa por ecografía Doppler y presencia o ausencia de SPT). Además, se identificaron los factores precipitantes o etiológicos de la TVPIF (condiciones médicas que generaban inmovilidad, síndromes de hipercoagulabilidad genéticos y adquiridos, y factores mecánicos locales).

Los procedimientos de TDC fueron realizados por miembros de la Sección de Radiología Intervencionista del Departamento de Imágenes Diagnósticas, en coordinación con el Servicio de Hematología y Cirugía Vascular Periférica. Los agentes trombolíticos (activadores tisulares del plasminógeno recombinantes) usados fueron alteplase (Actilyse ${ }^{\circledR}$ - Boehringer Ingelheim) o tenecteplase (Metalyse ${ }^{\circledR}$ - Boehringer Ingelheim), según su disponibilidad. Tras el ingreso de los pacientes por urgencias, se llevó a cabo el diagnóstico de TVP por ultrasonografía o angiografía por tomografía axial computarizada (angio-TAC); posteriormente, siguiendo un curso estándar, se inició anticoagulación con heparina de bajo peso molecular (HBPM).

De forma multidisciplinaria, se puso en común la elegibilidad para la TDC específica para cada caso. En los individuos analizados, se administró HBPM hasta al menos ocho horas antes del momento programado para la TDC; paralelamente, se expuso el paciente a una infusión continua de heparina no fraccionada (HNF) y su dosificación se controló según el nomograma de Raschke $^{30}$. Después de confirmar la hora estimada del procedimiento, se suspendió la infusión de HNF por un intervalo mínimo de cuatro horas respecto a esta. La TDC se efectuó en una sala de hemodinamia y bajo sedación asistida por el Servicio de Anestesiología. En todos los casos, el abordaje se realizó por vía posterior en la extremidad comprometida. Luego, previa asepsia y antisepsia e infiltración con lidocaína, una guía ecográfica y un sistema de micropunción, se cateterizó la vena poplítea y se avanzó un introductor vascular $5 \mathrm{Fr}$ (Maxxim Medical, Inc. Texas, EE.UU.), haciendo un estudio venográfico distal que confirmó la localización y longitud del trombo.

A continuación, se introdujo un catéter angulado junto a una guía hidrófila para avanzar a través de la carga trombótica hacia el segmento venoso proximal, llegando hasta la vena cava inferior (VCI) (donde se valoró la extensión y presencia de trombos flotantes). Luego de retirar dicho catéter, se avanzó sobre la guía fija un dispositivo de infusión Fountain 4Fr (Merit
Medical Systems Inc, EE.UU.) o una guía de infusión de Katzen (Boston Scientific, EE. UU.) hasta alcanzar la región proximal del trombo (vena iliaca). Una vez posicionados el sistema de infusión y el introductor vascular, se inició la infusión del agente trombolítico mediante un bolo seguido de un flujo continuo, en conjunto con HNF entre 300-500 UI/hora.

Tras fijar en la posición deseada el catéter y el introductor con apósitos estériles, los pacientes fueron trasladados a la unidad de cuidado intensivo para monitoreo hemodinámico y observación del sitio de punción. Se efectuaron controles diarios del perfil de hemostasia, así como venográficos en la sala de hemodinamia (cada 12 y 24 horas). Según el grado estimado de trombolisis en cada control, el catéter fue reposicionado para dirigir la infusión a determinada región en los casos en los que se obtuvo un resultado parcial. Entre cada control, se determinó continuar la dosificación trombolítica más la HFN, excepto cuando se evidenciaron los siguientes hallazgos: superar un tiempo de infusión mayor a 96 horas, lograr la lisis de más del 95\% del coágulo o no detectar aumento del porcentaje de trombolisis entre dos controles contiguos.

El diagnóstico de síndrome del May-Thurner (SMT) se hizo cuando se evidenció una lesión estenótica no trombótica en la venografía postrombolisis en la vena iliaca común, en el sitio esperado del cruce de los vasos arteriales, asociada a colateralización y derivación del flujo sanguíneo mediante ramas accesorias a este nivel. En caso de detectar la estenosis persistente mayor al $50 \%$ no atribuible al coágulo, se realizó una nueva caracterización venográfica en cuya base se decidió practicar una angioplastia por balón más colocación de un implante endovascular autoexpandible Wallstent ${ }^{\circledR}$ (Boston Scientific, EE. UU.) por la misma vía ya establecida. Este procedimiento se efectuó inmediatamente después de darse por concluida la TDC, en ausencia de complicaciones que requirieran una intervención urgente. Finalmente, la hemostasia manual local se aplicó al retirar los catéteres sobre el sitio de inserción y se restableció la anticoagulación plena con HBPM cuatro horas después de dicho procedimiento. La warfarina sódica se inició sólo hasta documentar la ausencia de complicaciones hemorrágicas asociadas a la TDC.

El seguimiento a corto y a largo plazo se realizó durante la hospitalización antes de concluir el primer 
mes, y se repitió en los siguientes seis meses tras la TDC en el ámbito ambulatorio. La valoración de los pacientes estuvo a cargo de especialistas en hematología y cirugía vascular periférica. Crónicamente, se estableció el uso de MCG y la terapia de anticoagulación con warfarina, controlando su dosificación según el cociente internacional normalizado (INR, por su sigla en inglés).

\section{Resultados}

\section{Características de los pacientes incluidos}

El estudio incluyó cuatro pacientes con una mediana de edad de 58 años (rango 35-70) y dominancia del sexo femenino (75\%). La mediana de duración de los síntomas fue 3.5 días (rango, 1-8) y la presentación clínica incluyó edema y dolor en todos los casos, mientras que cianosis y congestión venosa en menor proporción (75\%). Solo una paciente experimentó dificultad para la marcha asociada a debilidad. Ninguno refirió antecedentes personales de enfermedad arteriosclerótica o tromboembólica, y solo una tenía un familiar de estas condiciones (en primer grado). El único sujeto que no tuvo el SMT asociado a la TVPIF tenía como factor de riesgo significativo la inmovilidad (distrofia muscular con predominio de afectación sobre los miembros inferiores).

Al examen ultrasonográfico, los cuatro pacientes presentaron una TVPIF con extenso compromiso en tres o más venas del miembro inferior. A pesar de los días referidos de la sintomatología, dos presentaron trombos de aspecto subagudo. Los restantes tuvieron trombos de aspecto agudo. El procedimiento venográfico previo a la TDC no mostró en ningún caso la extensión ni la presencia de trombos flotantes en la $\mathrm{VCl}$.

\section{Trombolisis dirigida por catéter y desenlaces a corto plazo}

En ningún caso fue necesario colocar un filtro de VCI previo a la infusión. La mediana de duración del procedimiento fue 24.5 horas (rango, 20.5 a 28.5). En tres de los pacientes se utilizó tenecteplase, con una mediana de dosis infundida total mediana de $8.1 \mathrm{mg}$ (rango, 7 a 12.5). Solo en un paciente se utilizó alteplase con una dosis total de $30.5 \mathrm{mg}$. El éxito de la lisis de la carga trombótica medido en el último control venográfico fue del $90 \%$ (rango, $80 \%$ al 99\%) (figura 2). Al

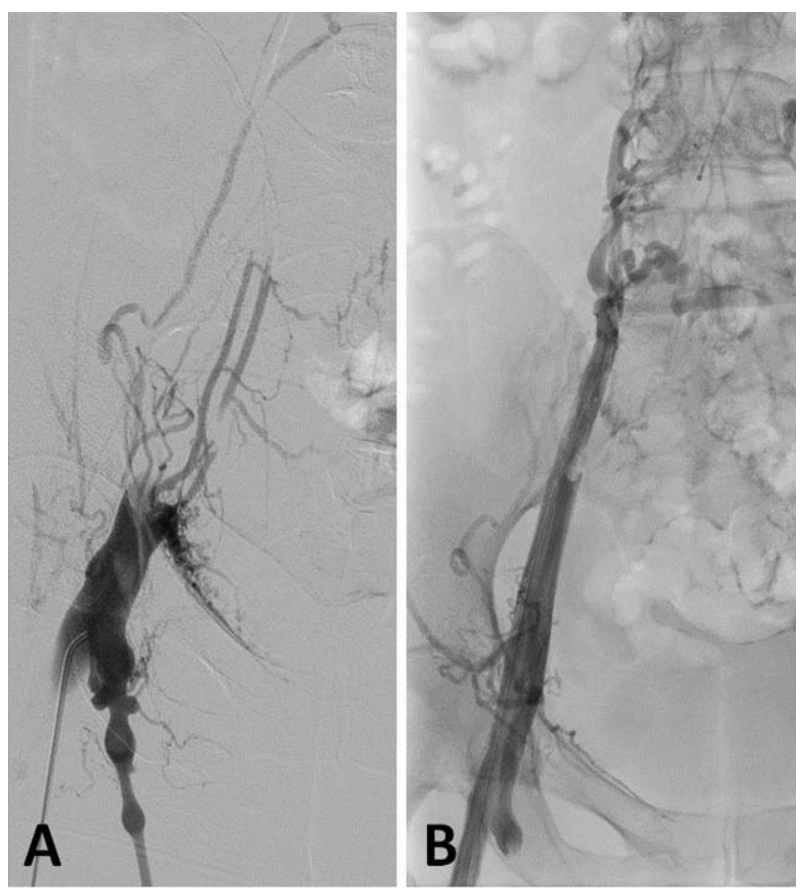

A. Se demuestra un defecto completo de llenamiento del medio de contraste a nivel de la vena iliaca común izquierda. Además, se observan proximalmente numerosas ramas colaterales. B. Posterior a la TDC, se observa la repermeabilización del segmento iliofemoral, con una mínima persistencia de la carga trombótica inicial; se registró un éxito trombolítico de más del 95\%. Además, se observa la estenosis luminal mayor al 50\% en las cercanías de la unión con la vena cava inferior, constituyendo el síndrome de May-Thurner.

Figura 2. Venografía del miembro inferior izquierdo de una de las pacientes incluidas en el estudio.

finalizar el tratamiento, se documentó una permeabilidad completa en dos sujetos, y parcial en el resto. En tres de los pacientes se presentaron algunos episodios hemorrágicos menores (no requirieron intervenciones hemostáticas) y gingivorragia. En la otra paciente se presentó una anemización aguda, que, aunque requirió transfusión de glóbulos rojos (sin identificarse en las imágenes hematomas de importancia), no tuvo repercusión adversa adicional. La mediana de días de hospitalización fue de 7 (rango, 4-9), siendo de mayor duración en los casos en los que hubo hemorragia. En ningún caso se presentó tromboembolismo pulmonar sintomático. A los tres casos diagnosticados como SMT (figura 3) se les practicó exitosamente la angioplastia y colocación de stent, logrando una permeabilidad final mayor al $80 \%$.

\section{Desenlaces a largo plazo}

La mediana de seguimiento después de la TDC fue de ocho meses (rango, 6-11), sin encontrar ninguna sintomatología de los componentes del SPT en el puntaje de Villalta. El control de la permeabilidad 

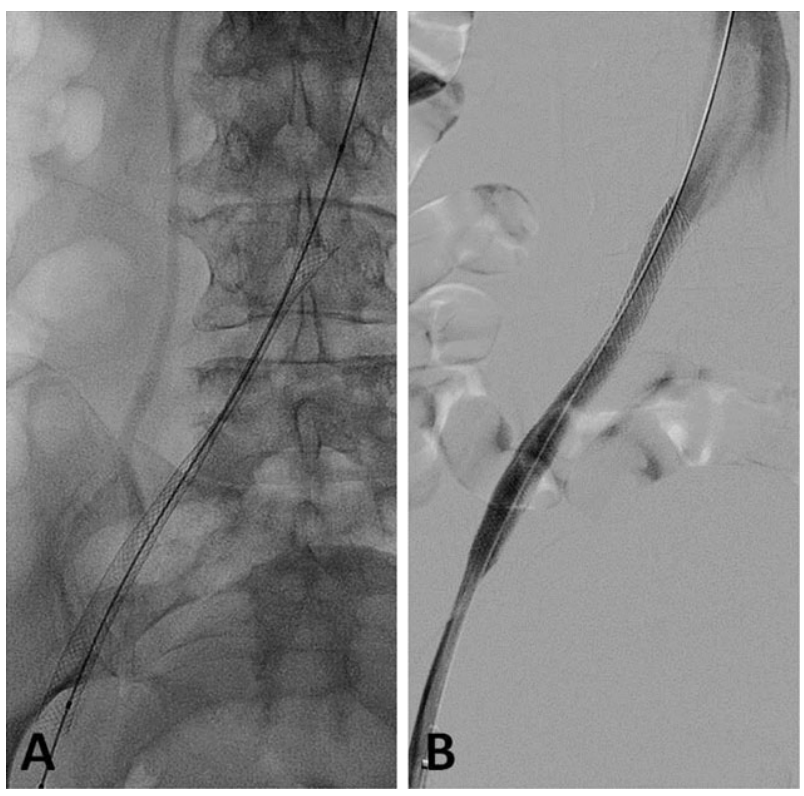

A. Se muestra el stent expandido automáticamente sobre el segmento venoso, exhibiendo una zona estenosada en más del $50 \%$ de la luz al nivel del sitio esperado del cruce de los vasos arteriales. B. Posterior a la expansión del stent mediante el balón, se observa la recuperación de la perme Bilidad del segmento linefemoral, con paso del medio de contraste hasta la vena cava inferior.

Figura 3. Angioplastia del segmento iliofemoral izquierdo con balón y colocación de un stent autoexpandible.

realizado por Doppler ultrasonográfico mostró la vigencia completa del sistema venoso iliofemoral en la extremidad comprometida en tres casos. En el cuarto paciente, se observó una recanalización parcial, sin que se hubiera perdido al menos la permeabilidad luminal del $80 \%$ inmediatamente obtenida tras la TDC. A la fecha de la última evaluación (junio del 2012), todos los pacientes se encontraban plenamente funcionales y acoplados a sus actividades diarias. Ninguno experimentó reestenosis del segmento venoso iliofemoral o recurrencia del TEV.

\section{Discusión}

Desde su descripción, las técnicas endovasculares diseñadas para el tratamiento de la TVPIF han experimentado notorios avances. El uso de catéteres para embolectomía por balón se constituyó como el primer intento terapéutico para manejar trombos de gran importancia clínica. Sin embargo, comprendía riesgos considerables, tales como la ruptura venosa, la denudación endotelial y la oclusión venosa temprana, debido a la hiperplasia de la íntima ${ }^{31}$. Por otra parte, en la década de 1960, se describieron varias experiencias y estudios clínicos europeos que propusieron la infusión sistémica
Tabla 1. Características de los pacientes trombolizados por TVP iliofemoral

\begin{tabular}{|c|c|c|c|c|}
\hline Característica & Paciente 1 & Paciente 2 & Paciente 3 & Paciente 4 \\
\hline \multicolumn{5}{|l|}{ Demografía } \\
\hline $\begin{array}{l}\text { Edad al procedimiento } \\
\text { (años) }\end{array}$ & 36 & 61 & 70 & 55 \\
\hline Sexo & $\mathrm{F}$ & $\mathrm{F}$ & $\mathrm{F}$ & M \\
\hline Factores precipitantes & $\mathrm{SMT}^{*}+\mathrm{ACO}^{* *}$ & SMT* & SMT* & $\begin{array}{l}\text { Distrofia } \\
\text { muscular }\end{array}$ \\
\hline $\begin{array}{l}\text { Duración de síntomas } \\
\text { (días) }\end{array}$ & 1 & 1 & 4 & 8 \\
\hline Lado comprometido & Izquierdo & Izquierdo & Izquierdo & Izquierdo \\
\hline Extensión del trombo & $\begin{array}{l}\text { Iliofemo- } \\
\text { rosafeno } \\
\text { poplítea }\end{array}$ & $\begin{array}{l}\text { lliofemo- } \\
\text { rosafeno } \\
\text { poplitea }\end{array}$ & $\begin{array}{l}\text { lliofemoropo- } \\
\text { plítea y tibial } \\
\text { posterior }\end{array}$ & $\begin{array}{l}\text { lliofemoro- } \\
\text { popliteay } \\
\text { pantorrilla }\end{array}$ \\
\hline $\begin{array}{l}\text { Característica } \\
\text { ecográfica del trombo }\end{array}$ & Subagudo & Agudo & Agudo & Subagudo \\
\hline Agente trombolítico & Tenecteplase & Alteplase & Tenecteplase & Tenecteplase \\
\hline Terapia adyuvante & $\begin{array}{c}\text { Angioplastia } \\
\text { + stent }\end{array}$ & $\begin{array}{l}\text { Angioplastia } \\
\text { con stent }\end{array}$ & $\begin{array}{l}\text { Angioplastia } \\
\text { con stent }\end{array}$ & No \\
\hline $\begin{array}{l}\text { Duración de la infusión } \\
\text { (h) }\end{array}$ & 28.5 & 20.5 & 25 & 24 \\
\hline $\begin{array}{l}\text { Dosis total de trombo- } \\
\text { lítico infundida (mg) }\end{array}$ & 8.13 & 30.5 & 12.5 & 7 \\
\hline
\end{tabular}

\begin{tabular}{|l|c|c|c|c|}
\hline \multicolumn{3}{|l|}{ Desenlaces tempranos (menos de 1 mes) } \\
\hline $\begin{array}{l}\text { Tiempo de hospitali- } \\
\text { zación posterior a la } \\
\text { trombolisis (días) }\end{array}$ & 7 & 8 & 9 & 4 \\
\hline Permeabilidad final (\%) & 85 & 99 & 95 & 80 \\
\hline Complicaciones & Gingivorragia & $\begin{array}{c}\text { Hematoma } \\
\text { poplíteo }\end{array}$ & $\begin{array}{c}\text { Hematomas } \\
\text { y equimosis }\end{array}$ & No \\
\hline Sangrado menor & No & $\begin{array}{c}\text { Anemización } \\
\text { y reque- } \\
\text { rimiento } \\
\text { transfusional }\end{array}$ & No \\
\hline Sangrado mayor & No & No & No & No \\
\hline $\begin{array}{l}\text { Embolismos pulmona- } \\
\text { res sintomáticos }\end{array}$ & No & & \\
\hline
\end{tabular}

\begin{tabular}{|l|c|c|c|c|}
\hline \multicolumn{2}{|l|}{ Desenlaces a largo plazo (más de 6 meses) } \\
\hline $\begin{array}{l}\text { Tiempo al último } \\
\text { control (meses) }\end{array}$ & 6 & 10 & 11 & 6 \\
\hline $\begin{array}{l}\text { Conclusión } \\
\text { ecografía Doppler }\end{array}$ & Normal & Normal & Normal & $\begin{array}{c}\text { Recanaliza- } \\
\text { ción parcial } \\
\text { sobre la vena } \\
\text { iliaca externa. } \\
\text { Sin reflujo. }\end{array}$ \\
\hline $\begin{array}{l}\text { Eventos tromboem- } \\
\text { bólicos recurrentes o } \\
\text { reestenosis }\end{array}$ & No & No & No & No \\
\hline $\begin{array}{l}\text { Síndrome } \\
\text { postrombótico }\end{array}$ & No & No & No & No \\
\hline
\end{tabular}

* Síndrome de May-Thurner

** Anticonceptivos orales

de agentes trombolíticos (principalmente estreptoquinasa) para diluir el coágulo y obviar la intervención quirúrgica del sistema venoso ${ }^{15,32}$.

Un metaanálisis de estos estudios determinó la eficacia comprobada de la estrategia al obtener con mayor frecuencia la lisis completa del coágulo y el mejoramiento de la permeabilidad venosa tanto a corto como a largo plazo. De igual forma, la incidencia de SPT a largo plazo se redujo a la mitad de los casos (RR 0.66, IC95\% 0.47-0.94). No obstante, estos beneficios se vieron 
limitados como consecuencia de una tasa significativa de complicaciones hemorrágicas (RR 1.73, IC95\% 1.042.88), incluyendo la más temida, la intracraneana ${ }^{15}$. La conclusión específica de dicho estudio integrativo fue un avance en la optimización de la terapia trombolítica; a medida que se caracterizaron los riesgos asociados y se afinaron los criterios de selección de los pacientes candidatos, se obtuvo una menor incidencia de complicaciones y mortalidad'5. Es por esto por lo que los criterios actuales para la terapia trombolítica se rigen sobre el riesgo de sangrado asociado a la intervención y se encuentran listados en la tabla 2.

No fue sino hasta 1994 cuando Semba y colaboradores publicaron el primer registro de una serie de pacientes tratados con TDC para TVPIF, justificando la concentración local del trombolítico mediante un catéter en homologación a los resultados observados en la lisis de coágulos arteriales ${ }^{33}$. Desde esa fecha, se han publicado numerosos reportes preliminares y conclusivos de casos y series, incluyendo al menos cinco estudios observacionales prospectivos ${ }^{10,11,33-35}$ y tres experimentos clínicos controlados con asignación aleatoria (ECA) ${ }^{36-38}$. El análisis estratificado de uno de los ECA $^{36}$ realizado por Watson y colaboradores ${ }^{15}$ concluyó que el uso de estreptoquinasa para la TDC resultó en una mayor probabilidad de lisis temprana del coágulo, sin diferencias en la tasa de presentación de fenómenos embólicos pulmonares. Cabe resaltar que, durante todo el seguimiento hasta los seis meses del estudio, no se

Tabla 2. Contraindicaciones absolutas y relativas para la TDC en TVPIF

\begin{tabular}{|l|l|}
\multicolumn{1}{|c|}{$\begin{array}{c}\text { Contraindicaciones } \\
\text { absolutas }\end{array}$} & \multicolumn{1}{c|}{ Contraindicaciones relativas } \\
\hline $\begin{array}{l}\text { Enfermedad intracraneana } \\
\text { estructural/anatómica }\end{array}$ & $\begin{array}{l}\text { Hipertensión arterial no controlada (presión sistólica > } \\
180 \mathrm{~mm} \text { Hg y/o presión diastólica > } 110 \mathrm{~mm} \text { Hg) }\end{array}$ \\
\hline $\begin{array}{l}\text { Historial de hemorragia intra- } \\
\text { craneana }\end{array}$ & Sangrado reciente, no intracraneano \\
\hline $\begin{array}{l}\text { Accidente cerebro-vascular de } \\
\text { tipo isquémico en los últimos } \\
\text { tres meses }\end{array}$ & Cirugía reciente \\
\hline Sangrado activo & Procedimientos invasivos recientes \\
\hline $\begin{array}{l}\text { Neurocirugía (cerebral o espinal) } \\
\text { reciente }\end{array}$ & $\begin{array}{l}\text { Accidente cerebro-vascular de tipo isquémico hace más } \\
\text { de tres meses }\end{array}$ \\
\hline \multirow{5}{*}{ Diátesis hemorrágica } & Anticoagulación activa (p. ej. con warfarina) \\
\cline { 2 - 3 } & Resucitación cardiopulmonar traumática \\
\cline { 2 - 3 } & Pericarditis o derrame pericárdico \\
\cline { 2 - 3 } & Retinopatía diabética \\
\cline { 2 - 3 } & Embarazo \\
\cline { 2 - 3 } & Edad $>75$ años \\
\cline { 2 - 3 } & Bajo peso corporal $(<60 \mathrm{~kg})$ \\
\cline { 2 - 3 } & Sexo femenino \\
\cline { 2 - 3 } & Raza negra \\
\hline
\end{tabular}

Fuente: tomado de Kearon C, AkI EA, Comerota AJ, Prandoni P, Bounameaux H, Goldhaber SZ, et al. Antithrombotic therapy for VTE disease: Antithrombotic Therapy and Prevention of Thrombosis, 9th ed: American College of Chest Physicians Evidence-Based Clinical Practice Guidelines. Chest 2012;141(2 Suppl):e4195-945. registraron casos de hemorragia intracraneana o muerte en ninguno de los dos brazos.

A largo plazo, el uso de la TDC resultó en una mayor probabilidad de control de la obstrucción, así como de recuperación de la funcionalidad venosa ${ }^{15}$. No obstante, este metaanálisis deberá actualizarse en un futuro cercano, como consecuencia de la publicación reciente de los resultados del resto de los ECA. El estudio Cavent, llevado a cabo en Noruega (NCT00251771), aleatorizó 209 pacientes con un primer episodio TVPIF a recibir el tratamiento estándar o este más la TDC utilizando alteplase en infusión continua. A 24 meses de seguimiento, se observó una reducción absoluta del riesgo de SPT en casi el 15\% (IC95\% 0.2-27.9), registrando una frecuencia del $41 \%$ en el grupo de TDC ${ }^{37}$. La permeabilidad iliofemoral también se recuperó significativamente en el grupo de TDC, en el que se registraron 3 y 5 complicaciones hemorrágicas mayores y clínicamente significativas, respectivamente.

Ningún paciente murió o tuvo embolismos durante la realización de la TDC y la incidencia de fenómenos TEV recurrentes en ambos grupos no resultó diferencialmente positiva. Tales resultados han sido controvertidos debido a una diferencia en la frecuencia de uso de MCG y por el tiempo bajo anticoagulación en INR terapéutico entre ambos grupos ${ }^{39}$. Por su parte, el estudio Attract (NCT00790335), que involucra una estrategia trombolítica farmacomecánica, aún se encuentra en curso $^{38}$. En el presente reporte, a pesar del limitado número de pacientes, pudimos observar características clínicas y demográficas similares con aquellas reportadas en estos trabajos. Así mismo, los resultados medidos a corto y a largo plazo fueron similares, logrando y manteniendo la permeabilidad venosa.

En el año 2003, las sociedades norteamericanas y europeas de radiología intervencionista convinieron mediante un consenso que el objetivo principal de la terapia trombolítica debería ser la obtención de desenlaces clínicos, los cuales prevalecerían sobre los físicos o fisiológicos ${ }^{27}$. Por tal motivo, resulta coherente hacer énfasis en la detección del impacto de la TDC sobre la génesis del SPT como desenlace principal a largo plazo. Pese a esto, los ECA han obtenido magnitudes heterogéneas de la reducción del riesgo de SPT. A manera de ejemplo, para la determinación del impacto sobre el SPT, Elsharawy y colaboradores detectaron un beneficio cuantificado como función venosa normal con un 
RR 0.16 (IC95\% 0.04-0.62) para los pacientes tratados con TDC sobre los que fueron tratados convencionalmente a los seis meses de seguimiento ${ }^{15,36}$.

Por otro lado, el estudio Cavent determinó el impacto de la TDC utilizando una escala específica para SPT, basada en síntomas y signos, tras dos años de seguimiento; sin embargo, este mismo desenlace no fue diferente para ambos grupos cuando fue medido a los seis meses después de la TDC. Lo anterior demuestra la variabilidad existente en la evidencia para la estimación de la eficacia de la TDC a largo plazo. Una posible explicación para este hecho radica en el uso de diversas escalas para establecer el diagnóstico de SPT, las cuales se han implementado según la experiencia y conveniencia de cada grupo. En la literatura sobre SPT, se han empleado al menos seis escalas, de las que tres se desarrollaron específicamente para su diagnóstico (Brandjes, Ginsberg y Villalta) ${ }^{40,41}$.

Estas escalas se diferencian fundamentalmente en el espectro clínico que consideran, así como en los rangos temporales para la evaluación de signos y síntomas. Ante este panorama, en el 2009, la International Society on Thrombosis and Haemostasis ${ }^{42}$ recomendó la estandarización del puntaje de Villalta para los estudios clínicos cuyo objetivo sea establecer el diagnóstico, determinar la severidad y valorar la evolución de dicha patología. Los componentes que considera ese puntaje se resumen en la tabla $3^{37,43}$ (ver guía visual disponible en línea como anexo electrónico). La estandarización de estos esfuerzos ayudará a consolidar el impacto de la TDC sobre la génesis del SPT a largo plazo.

Adicional a los problemas que surgen al determinar los desenlaces asociados a la TDC, tampoco existe un estándar procedimental universalmente aceptado. Como consecuencia de la definición técnica de cada

Tabla 3. Signos y síntomas que componen el puntaje de Villalta para el diagnóstico y seguimiento del SPT

\begin{tabular}{|c|c|}
\hline Síntomas* & Signos clínicos* \\
\hline Dolor & Edema pretibial \\
\hline Calambres & Induración de la piel \\
\hline Pesadez de la extremidad & Hiperpigmentación \\
\hline Parestesias & Eritema \\
\hline Prurito & Ectasia venosa \\
\hline Ulceración** & Dolor a la compresión del tobillo \\
\hline \multicolumn{2}{|c|}{$\begin{array}{l}\text { * Cada síntoma o signo se gradúa de la siguiente forma: ausente } 0 \text { puntos; leve } 1 \text { punto; } \\
\text { moderado } 2 \text { puntos; severo } 3 \text { puntos. } \\
\text { ** Se gradúa únicamente como presente o ausente. } \\
\text { La escala va de } 0 \text { a } 33 \text { puntos totales. La sumatoria de los puntos define: } \\
\text { Diagnóstico de PTS: } \geq 5 \text { puntos o presencia de al menos una úlcera venosa } \\
\text { Severidad del PTS: leve 5-9 puntos, moderado } 10-14 \text { puntos, severo } \geq 15 \text { puntos o presencia } \\
\text { de al menos una úlcera venosa }\end{array}$} \\
\hline
\end{tabular}

grupo de manera local, se ha limitado la posibilidad de realizar contrastes directos. El único esfuerzo de estandarización de la TDC se llevó a cabo en el 2003 por un consenso multidisciplinario entre especialistas de Europa y Norteamérica ${ }^{27}$. Siguiendo este patrón, en nuestro centro, se ha intentado efectuar homogéneamente el procedimiento para todos los casos.

Cabe resaltar como limitante la disponibilidad de los trombolíticos, lo que obligó al uso del tenecteplase en la mayoría de nuestros casos. Si bien gran parte de los estudios han utilizado alteplase, hasta la fecha no existe ningún ECA que haya comparado directamente la eficacia de los diversos agentes trombolíticos para esta indicación. A pesar de ello, algunas series retrospectivas han resaltado que los tiempos de infusión, las tasas del éxito trombolítico y las complicaciones son equiparables para la uroquinasa, el alteplase y el reteplase ${ }^{44,45}$. El Consenso Colombiano de Trombolisis ofrece detalles específicos de la farmacocinética y eficacia de cada agente trombolítico disponible en el país ${ }^{46}$.

Nuestra experiencia en TDC con tenecteplase para TVPIF, si bien parece incipiente, ha resultado en un beneficio clínico considerable y congruente con lo reportado en la literatura hasta la fecha. No obstante, pocos grupos colombianos han reportado su experiencia con TDC en el manejo de la TVPIF ${ }^{47,48}$. Patiño y colaboradores describieron el uso de TDC con estreptoquinasa (principalmente) y uroquinasa para pacientes con trombosis venosa y arterial en el Hospital San Vicente de Paúl por más de tres años. Para los casos de TVP, concluyeron una tasa de éxito trombolítico del $57 \%{ }^{48}$. Sin embargo, fue evidente una gran heterogeneidad clínica entre los pacientes de este reporte (por su localización, tiempo de evolución -que incluso varió entre 1 y 540 días de síntomas-, terapias concomitantes y factores de riesgo), lo que dificultó la proposición para determinantes para el éxito trombolítico. Es importante anotar que la frecuencia global de complicaciones asociadas a la TDC fue del $40 \%$, siendo las más frecuentes las complicaciones hemorrágicas (menores en un 33\% y mayores en el $13 \%)^{48}$.

Directamente asociadas a la TDC, en otros estudios, la tasa de complicaciones hemorrágicas mayores se ha reportado entre el $5 \%$ y $11 \%{ }^{13}$, incluyendo la hemorragia intracraneana $(<1 \%)$, el hematoma retroperitoneal (1\%) y los sangrados músculo-esqueléticos, 
genitourinarios y gastrointestinales $(3 \%)^{10}$. Por otro lado, las complicaciones hemorrágicas menores han sido las más frecuentes (16\%), ocurriendo la mitad en el sitio de punción, por lo que la guía ecográfica durante la colocación del catéter resulta fundamental10. Concordante con esta información, las frecuencias y tipo de estos desenlaces en nuestro reporte fueron similares.

La ventana de tiempo desde la presentación de la sintomatología que se considera válida para la realización de la TDC ha sido ampliamente discutida, variando desde los 10 hasta los 21 días $^{26}$. En varios estudios, se determinó que la cronicidad del coágulo determina la probabilidad del éxito trombolítico, como consecuencia de su remodelación ${ }^{10,33}$. El reciente Consenso del American College of Chest Physicians ha definido como criterio para la realización de la TDC la presentación de TVPIF, con una sintomatología inferior a los 14 días, en pacientes que tengan una expectativa de vida mayor o igual a un año, y con un bajo riesgo de sangrado ${ }^{26}$

Estos criterios están en acuerdo con los establecidos por el Consenso Colombiano de Trombolisis, considerando además como necesaria la individualización de cada caso ${ }^{46}$. En la serie de la Fundación Santa Fe de Bogotá, todos los pacientes estaban previamente asintomáticos y la mediana de establecimiento del cuadro vascular fue inferior a dos semanas. Adicionalmente, quienes tuvieron un trombo de características subagudas fueron los mismos que lograron una permeabilidad parcial después de la trombolisis. Este hallazgo permite plantear una hipótesis a favor de la posibilidad de utilizar la trombolisis en sujetos con obstrucciones subagudas.

La necesidad de implantar filtros de vena cava superior (FVCS) como paso previo a la realización de la TDC ha sido una práctica debatida, a excepción de la prevención de eventos embólicos en pacientes con trombosis y contraindicaciones para la anticoagulación ${ }^{26}$. Las series de casos de pacientes llevados a TDC han exhibido seguimientos libres de eventos embólicos pulmonares hasta por tres años, incluso sin haber requerido la colocación de FVCS ${ }^{49}$. Debido a su baja ocurrencia, la diferenciación definitiva entre los eventos tromboembólicos pulmonares originados durante la TDC o por desprendimiento de los trombos en proceso de recanalización no ha sido factible. Por el momento, resulta necesario considerar el riesgo embólico específico asociado al procedimiento en cada caso particular ${ }^{16}$. En nuestro estudio, esta consideración no fue necesaria para ningún paciente, y fue constatado, pues no se evidenció ningún evento embólico pulmonar tras la TDC ${ }^{38}$.

Se han descrito varios factores de riesgo de tipo mecánico que promueven la génesis de trombos sobre el eje iliofemoral. Estos comprenden los tumores pélvicos, los osteofitos, la retención urinaria crónica, los aneurismas arteriales iliacos, la endometriosis, el embarazo y las masas uterinas. No obstante, es la compresión extrínseca de la vena iliaca común izquierda por la respectiva arteria iliaca común la causa mecánica más frecuente ${ }^{50}$. Este síndrome fue descrito por May y Thurner (SMT), en 1956, como el hallazgo de tres variedades de crecimientos o espolones intraluminales localizados dentro de la vena iliaca común izquierda en el $22 \%$ de 430 estudios anatómicos cadavéricos ${ }^{51}$.

Se postuló que este fenómeno era causado por un proceso fisiopatológico pulsátil de frotamiento crónico de la vena por parte de la arteria contra el cuerpo vertebral lumbar. Crónicamente, esto resultaba en una proliferación local de la íntima, que, sumada a un limitado retorno venoso, promovía la estasis sanguínea y, en últimas, la trombosis ${ }^{52,53}$. Como consecuencia y evidencia de la obstrucción al flujo fisiológico, Cockett además caracterizó la formación de ramas venosas colaterales que permiten su derivación y adaptación para restaurar el retorno venoso a la vena cava ${ }^{53}$.

En el 2000, Baron y colaboradores describieron el uso de la angioplastia por balón más la colocación de un stent como una opción factible para recuperar y mantener la permeabilidad venosa en los pacientes con SMT ${ }^{54}$. De forma similar, en nuestro país, García y colaboradores difundieron su experiencia con la terapia endovascular y anticoagulación para el manejo de 17 pacientes con SMT, con o sin trombosis asociada ${ }^{55}$. La permeabilidad de los stents en diversos puntos temporales a largo plazo fue reportada en el $94.1 \%$ de los casos, justificando el papel fundamental del stent en la prevención de la reestenosis. Aun así, este reporte careció de información de seguridad asociada al dispositivo.

En contraste, algunos estudios sugirieron que la estrategia estándar de manejo con TDC sin la colocación del stent para tratar los casos de SMT y TVPIF resultó en una frecuencia de recurrencia trombótica en más del $70 \%$ de los $\operatorname{casos}^{52,56}$. Más aún, se ha definido que la probabilidad de lograr la recanalización 
de un evento trombótico en el segmento iliofemoral sujeto a dicho síndrome compresivo a largo plazo es menor a comparación de los casos no afectados por la compresión ${ }^{57}$. Por lo tanto, las consecuencias del SMT implican pacientes con fenómenos trombóticos recurrentes y/o reestenosis que experimentan una importante morbilidad venosa crónica. La evidencia anterior justificó el uso de una terapia trombolítica y mecánica conjunta.

A la fecha, varias series de casos con al menos cinco pacientes sometidos a dicha estrategia ${ }^{52,56,58-61}$ reportaron entre el $87 \%$ y $100 \%$ de éxito trombolítico inmediato; algunos han demostrado la conservación de la permeabilidad de la región iliofemoral sostenida incluso hasta un año después de las TDC en más del $80 \%{ }^{52,58-61}$. La información a tiempos de seguimientos más largos es limitada, pero fue preliminarmente reportada como el mantenimiento de la permeabilidad venosa en más del $95 \%$ de los casos a dos años tras la TDC ${ }^{61}$. Como soporte adicional, pareciera no mantenerse la permeabilidad cuando no se coloca un stent tras la angioplastia ${ }^{52}$. No obstante, se ha sugerido que los stents venosos podrían inducir recurrencias de TVPIF a largo plazo como consecuencia de la presentación de un bajo flujo luminal62; se requerirán estudios con mayor tiempo de seguimiento que puedan refutar el aparente beneficio clínico que estos representan en la actualidad.

A pesar del limitado nivel de evidencia que defiende esta estrategia, se trata de la práctica clínica con mayor justificación. En nuestro medio, ha sido relativamente conocido el uso conjunto de las estrategias farmacomecánicas para el tratamiento de la TVPIF ${ }^{47}$, logrando importantes tasas de mantenimiento de la permeabilidad hasta por dos años de seguimiento, a expensas de complicaciones hemorrágicas menores en el sitio de punción. En este reporte, una proporción de pacientes tuvo SMT y fue sometida a la estrategia conjunta, obteniendo como resultado la permeabilidad sostenida a seis meses y la ausencia de síntomas del SPT. En ningún caso, hubo reestenosis del stent o recurrencia de la trombosis. Lo anterior resultó consistente con la experiencia reportada en la literatura.

En la actualidad en nuestro medio, el uso de la TDC no ha podido extenderse sistemáticamente. Diversos factores, tales como la necesidad de un equipo especializado en radiología intervencionista, una unidad de cuidados intensivos, los costos y los equipos que implica el entrenamiento y el procedimiento, han estado en contra del ofrecimiento de esta alternativa a los pacientes que más se podrían beneficiar de ella. Además, debido a que en su gran mayoría estos pacientes son atendidos de manera inicial por los servicios de urgencias, quienes no atenderán las consecuencias crónicas producto de la IVC, existe un desconocimiento general del impacto de la TDC ${ }^{38}$.

En el desarrollo tecnológico del campo, la TDC ha dado paso al diseño de sistemas de trombolisis farmacomecánica simultánea. Su fundamento radica en el asilamiento del trombo entre los segmentos proximal y distal, permitiendo suministrar y concentrar el trombolítico en el sitio que requiere su acción, y, al mismo tiempo, combinando los métodos físicos y químicos para lograr una trombolisis con mayor eficacia. Estos métodos incluyen el uso de catéteres con alambres de dispersión de rotación sinusoidal, denominado Trellis-8 (Bacchus Vascular Inc, California, EE. UU. ${ }^{63}$; un sistema de propulsión e inducción de flujos negativos de solución salina, denominado Angiojet (Possis Medical Inc, Minnesota, EE. UU. ${ }^{64}$; o un sistema combinado de ultrasonido de alta frecuencia y baja energía para promover la lisis mecánica, denominado catéter Lysus (Ekos, Washington, EE. UU.) ${ }^{65}$.

Todos estos nuevos sistemas han ofrecido menores tiempos y dosis durante el procedimiento trombolítico, la optimización de la lisis, incluso para los coágulos más crónicos, y menores costos derivados del seguimiento y la atención de complicaciones embólicas y hemorrágicas con la técnica estándar. Nuevos agentes trombolíticos también se encuentran en desarrollo, tales como el alfimeprase (Nuvelo, California, EE. UU.) y la plasmina humana purificada, que ofrecen una acción trombolítica más controlada a nivel local ${ }^{66}$. Hasta el momento, estas estrategias no se han evaluado en ECA y su efectividad clínica frente a la estrategia estándar para la TDC a largo plazo debe ser establecida ${ }^{16}$. Los resultados del estudio Attract darán nuevas luces en este aspecto.

\section{Conclusiones}

La experiencia inicial de TDC para pacientes con TVPIF aguda en la FSFB, siguiendo un modelo de grupo multidisciplinario que incluye hematología, radiología intervencionista y cirugía vascular periférica, demostró 
que el procedimiento es factible de realizar. A corto y largo plazo, este procedimiento ofreció resultados similares a los reportados en la literatura. Es fundamental reconocer que la seguridad de la TDC reposa sobre una adecuada selección individual de los pacientes candidatos. Adicionalmente, el SMT asociado a TVPIF es factible de ser manejado mediante una estrategia endovascular y farmacológica conjunta (TDC más angioplastia y colocación de stent), manteniendo la permeabilidad venosa y previniendo la insuficiencia crónica, característica de la historia natural de esta condición.

\section{Agradecimientos}

Los autores desean agradecer a Jenny Machetá, referencista de la Biblioteca de Medicina de la Universidad de los Andes, por su asistencia en la obtención de los textos completos utilizados en la discusión de este artículo. También agradecen a Angélica González Rodríguez, del Comité Editorial de la Revista Colombiana de Radiología, por su asistencia en la obtención de los reportes de experiencias locales en TDC.

\section{Referencias}

1. MeSH Database. Venous thrombosis. Bethesda, MD, EE. UU.: National Center for Biotechnology Information, U.S. National Library of Medicine 1999 [consultado el 05/jul/2012]. Disponible en: <http://www.ncbi.nlm.nih.gov/mesh/68020246>.

2. Center for Disease Control and Prevention. Venous thromboembolism in adult hospitalizations - United States, 2007-2009. MMWR Morb Mortal Wkly Rep. 2012;61:401-4.

3. White RH. The epidemiology of venous thromboembolism. Circulation. 2003;107(23 Suppl 1):14-8.

4. Goldhaber SZ, Bounameaux H. Pulmonary embolism and deep vein thrombosis. Lancet. 2012;379(9828):1835-46.

5. Kearon C. Natural history of venous thromboembolism. Circulation. 2003;107(23 Suppl 1):122-30

6. Killewich LA, Macko RF, Cox K, Franklin DR, Benjamin ME, Lilly MP, et al. Regression of proximal deep venous thrombosis is associated with fibrinolytic enhancement. J Vasc Surg. 1997;26(5):861-8.

7. van Ramshorst $B$, van Bemmelen PS, Hoeneveld $H$, Faber JA, Eikelboom BC. Thrombus regression in deep venous thrombosis. Quantification of spontaneous thrombolysis with duplex scanning. Circulation. 1992;86(2):414-9.

8. Piovella F, Crippa L, Barone M, Vigano D'Angelo S, Serafini S, Galli L, et al. Normalization rates of compression ultrasonography in patients with a first episode of deep vein thrombosis of the lower limbs: association with recurrence and new thrombosis. Haematologica. 2002;87(5):515-22.

9. Delis KT, Bountouroglou D, Mansfield AO. Venous claudication in iliofemoral thrombosis: long-term effects on venous hemodynamics, clinical status, and quality of life. Ann Surg. 2004;239(1):118-26

10. Mewissen MW, Seabrook GR, Meissner MH, Cynamon J, Labropoulos N, Haughton SH. Catheter-directed thrombolysis for lower extremity deep venous thrombosis: report of a national multicenter registry. Radiology. 1999;211(1):39-49.

11. AbuRahma AF, Perkins SE, Wulu JT, Ng HK. lliofemoral deep vein thrombosis: conventional therapy versus lysis and percutaneous transluminal angioplasty and stenting. Ann Surg. 2001;233(6):752-60.

12. Kahn SR. The post-thrombotic syndrome. Hematology Am Soc Hematol Educ Program. 2010;2010:216-20.
13. Bergqvist $D$, Jendteg $S$, Johansen $L$, Persson $U$, Odegaard $K$. Cost of long-term complications of deep venous thrombosis of the lower extremities: an analysis of a defined patient population in Sweden. Ann Intern Med. 1997;126(6):454-7.

14. Akesson H, Brudin L, Dahlström JA, Eklöf B, Ohlin P, Plate G. Venous function assessed during a 5 year period after acute iliofemoral venous thrombosis treated with anticoagulation. Eur J Vasc Surg. 1990;4(1):43-8.

15. Watson LI, Armon MP. Thrombolysis for acute deep vein thrombosis. Cochrane Database Syst Rev. 2004;(4):CD002783.

16. Gogalniceanu P, Johnston CJ, Khalid U, Holt PJ, Hincliffe R, Loftus IM, et al. Indications for thrombolysis in deep venous thrombosis. Eur J Vasc Endovasc Surg. 2009;38(2):192-8.

17. Saharay M, Shields DA, Porter JB, Scurr JH, Coleridge Smith PD. Leukocyte activity in the microcirculation of the leg in patients with chronic venous disease. J Vasc Surg. 1997;26(2):265-73.

18. Schmid-Schonbein G. Molecular basis of venous insufficiency. En: Bergan JJ, editor. The vein book. Burlington, MA, EE. UU.: Elsevier; 2007. p. 67-79.

19. Kahn SR, Shrier I, Julian JA, Ducruet T, Arsenault L, Miron MJ, et al. Determinants and time course of the postthrombotic syndrome after acute deep venous thrombosis. Ann Intern Med. 2008;149(10):698-707.

20. Ziegler S, Schillinger M, Maca TH, Minar E. Post-thrombotic syndrome after primary event of deep venous thrombosis 10 to 20 years ago. Thromb Res. 2001;101(2):23-33.

21. Saarinen J, Kallio $T$, Lehto $M$, Hiltunen $S$, Sisto $T$. The occurrence of the post-thrombotic changes after an acute deep venous thrombosis. A prospective two-year follow-up study. J Cardiovasc Surg (Torino). 2000;41(3):441-6.

22. Prandoni $P$, Lensing $A W$, Prins $M H$, Frulla $M$, Marchiori $A$, Bernardi $E$, et al. Below-knee elastic compression stockings to prevent the post-thrombotic syndrome: a randomized, controlled trial. Ann Intern Med. 2004;141(4):249-56.

23. Brandjes DP, Büller HR, Heijboer $H$, Huisman MV, de Rijk M, Jagt $H$, et al. Randomised trial of effect of compression stockings in patients with symptomatic proximal-vein thrombosis. Lancet. 1997;349(9054):759-62.

24. Kahn SR, Azoulay L, Hirsch A, Haber M, Strulovitch C, Shrier I. Effect of graduated elastic compression stockings on leg 
symptoms and signs during exercise in patients with deep venous thrombosis: a randomized cross-over trial. J Thromb Haemost. 2003;1(3):494-9.

25. Kahn SR, Shbaklo H, Lamping DL, Holcroft CA, Shrier I, Miron MJ, et al. Determinants of health-related quality of life during the 2 years following deep vein thrombosis. J Thromb Haemost. 2008;6(7):1105-12.

26. Kearon C, Akl EA, Comerota AJ, Prandoni $P$, Bounameaux $H$, Goldhaber SZ, et al. Antithrombotic therapy for VTE disease: Antithrombotic Therapy and Prevention of Thrombosis, 9th ed: American College of Chest Physicians Evidence-Based Clinical Practice Guidelines. Chest. 2012;141(2 Suppl):e419S-94S.

27. Working Party on Thrombolysis in the Management of Limb Ischemia. Thrombolysis in the management of lower limb peripheral arterial occlusion--a consensus document. J Vasc Interv Radiol. 2003;14(9 Pt 2):S337-49.

28. Lansing AM, Davis WM. Five-year follow-up study of iliofemoral venous thrombectomy. Ann Surg. 1968;168(4):620-8.

29. Vedantham S, Millward SF, Cardella JF, Hofmann LV, Razavi MK, Grassi CJ, et al. Society of Interventional Radiology position statement: treatment of acute iliofemoral deep vein thrombosis with use of adjunctive catheter-directed intrathrombus thrombolysis. J Vasc Interv Radiol. 2006;17(4):613-6.

30. Raschke RA, Gollihare B, Peirce JC. The effectiveness of implementing the weight-based heparin nomogram as a practice guideline. Arch Intern Med. 1996;156(15):1645-9.

31. Lin PH, Zhou W, Dardik A, Mussa F, Kougias P, Hedayati $N$, et al. Catheter-direct thrombolysis versus pharmacomechanical thrombectomy for treatment of symptomatic lower extremity deep venous thrombosis. Am J Surg. 2006;192(6):782-8

32. Robertson BR, Nilsson IM, Nylander G. Value of streptokinase and heparin in treatment of acute deep venous thrombosis. A coded investigation. Acta Chir Scand. 1968;134(3):203-8.

33. Semba CP, Dake MD. lliofemoral deep venous thrombosis: aggressive therapy with catheter-directed thrombolysis. Radiology. 1994;191(2):487-94.

34. Verhaeghe R, Stockx L, Lacroix H, Vermylen J, Baert AL. Catheter-directed lysis of iliofemoral vein thrombosis with use of rtPA. Eur Radiol. 1997:7(7):996-1001.

35. Bjarnason H, Kruse JR, Asinger DA, Nazarian GK, Dietz CA $J r$, Caldwell MD, et al. Iliofemoral deep venous thrombosis: safety and efficacy outcome during 5 years of catheter-directed thrombolytic therapy. J Vasc Interv Radiol. 1997;8(3):405-18

36. Elsharawy M, Elzayat E. Early results of thrombolysis vs anticoagulation in iliofemoral venous thrombosis. A randomised clinical trial. Eur J Vasc Endovasc Surg. 2002;24(3):209-14

37. Enden $T$, Haig $Y$, Klow NE, Slagsvold CE, Sandvik L, Ghanima $W$, et al. Long-term outcome after additional catheter-directed thrombolysis versus standard treatment for acute iliofemoral deep vein thrombosis (the CaVenT study): a randomised controlled trial. Lancet. 2012;379(9810):31-8.

38. Comerota AJ. The ATTRACT trial: rationale for early intervention for iliofemoral DVT. Perspect Vasc Surg Endovasc Ther. 2009;21(4):221-4.

39. Enden T, Kløw NE, Sandset PM. Catheter-directed thrombolysis for acute deep vein thrombosis--Authors' reply. The Lancet. 2012;379(9828):1786-7
40. Kahn SR. The post-thrombotic syndrome: progress and pitfalls. Br J Haematol. 2006:134(4):357-65.

41. Vedantham S, Grassi CJ, Ferral H, Patel NH, Thorpe PE, Antonacci VP, et al. Reporting standards for endovascular treatment of lower extremity deep vein thrombosis. J Vasc Interv Radiol. 2006;17(3):417-34

42. Kahn SR, Partsch $H$, Vedantham S, Prandoni P, Kearon C. Definition of post-thrombotic syndrome of the leg for use in clinical investigations: a recommendation for standardization. J Thromb Haemost. 2009;7(5):879-83.

43. Villalta S, Bagatella P, Piccioli A, Lensing AW, Prins MH, Prandoni P. Assessment of validity and reproducibility of a clinical scale for the postthrombotic syndrome. Haemostasis. 1994;24(suppl 1):157.

44. Sugimoto K, Hofmann LV, Razavi MK, Kee ST, Sze DY, Dake $M D$, et al. The safety, efficacy, and pharmacoeconomics of lowdose alteplase compared with urokinase for catheter-directed thrombolysis of arterial and venous occlusions. J Vasc Surg. 2003;37(3):512-7.

45. Grunwald MR, Hofmann LV. Comparison of urokinase, alteplase, and reteplase for catheter-directed thrombolysis of deep venous thrombosis. J Vasc Interv Radiol. 2004;15(4):347-52.

46. Red Nacional de Trombolisis. Otras indicaciones de tratamiento trombolítico. En: Castillo-Barrios GA, editor. Consenso Colombiano de Trombólisis y Terapias de Reperfusión. Bogotá: Ministerio de la Protección Social; 2008.

47. Hernández C, Gómez F, Mármol A, Escobar A, Duque J, Jiménez $A$, et al. Trombolisis selectiva y angioplastia con stent para el manejo de la trombolisis venosa profunda iliofemoral. Rev Colomb Cardiol. 2003;10(9):519.

48. Patiño JH, Cruz LA, León ME, Urbina CA, Cárdenas RA. Terapia fibrinolítica. Rev Colomb Radiol. 2003;14(4):1452-63.

49. Protack CD, Bakken AM, Patel N, Saad WE, Waldman DL, Davies MG. Long-term outcomes of catheter directed thrombolysis for lower extremity deep venous thrombosis without prophylactic inferior vena cava filter placement. J Vasc Surg. 2007;45(5):992-7.

50. Kibbe MR, Ujiki M, Goodwin AL, Eskandari M, Yao J, Matsumura J. Iliac vein compression in an asymptomatic patient population. J Vasc Surg. 2004;39(5):937-43.

51. May R, Thurner J. [A vascular spur in the vena iliaca communis sinistra as a cause of predominantly left-sided thrombosis of the pelvic veins]. Z Kreislaufforsch. 1956;45(23-24):912-22.

52. Kim JY, Choi D, Guk Ko Y, Park S, Jang Y, Lee do Y. Percutaneous treatment of deep vein thrombosis in May-Thurner syndrome. Cardiovasc Intervent Radiol. 2006;29(4):571-5.

53. Cockett FB, Thomas ML, Negus D. Iliac vein compression.--Its relation to iliofemoral thrombosis and the post-thrombotic syndrome. BMJ. 1967;2(5543):14-9.

54. Baron HC, Shams J, Wayne M. Iliac vein compression syndrome: a new method of treatment. Am Surg. 2000;66(7):653-5.

55. García LF, Francisco A, Franco CA, Lechter A. Manejo endovascular en pacientes con síndrome de compresión iliocava: experiencia en 17 casos. Rev Colomb Cardiol. 2006;13:23-30.

56. Husmann MJ, Heller G, Kalka C, Savolainen H, Do DD, Schmidli J, et al. Stenting of common iliac vein obstructions combined with regional thrombolysis and thrombectomy in acute deep vein thrombosis. Eur J Vasc Endovasc Surg. 2007;34(1):87-91 
57. Fraser DG, Moody AR, Morgan PS, Martel A. Iliac compression syndrome and recanalization of femoropopliteal and iliac venous thrombosis: a prospective study with magnetic resonance venography. J Vasc Surg. 2004;40(4):612-9.

58. O'Sullivan GJ, Semba CP, Bittner CA, Kee ST, Razavi MK, Sze DY, et al. Endovascular management of iliac vein compression (May-Thurner) syndrome. J Vasc Interv Radiol. 2000;11(7):82336.

59. Patel NH, Stookey KR, Ketcham DB, Cragg AH. Endovascular management of acute extensive iliofemoral deep venous thrombosis caused by May-Thurner syndrome. J Vasc Interv Radiol. 2000;11(10):1297-302.

60. Heijmen RH, Bollen TL, Duyndam DA, Overtoom TT, Van Den Berg JC, Moll FL. Endovascular venous stenting in May-Thurner syndrome. J Cardiovasc Surg (Torino). 2001;42(1):83-7.

61. Kwak HS, Han YM, Lee YS, Jin GY, Chung GH. Stents in common iliac vein obstruction with acute ipsilateral deep venous thrombosis: early and late results. J Vasc Interv Radiol. 2005;16(6):815-22
62. Patterson BO, Hinchliffe R, Loftus IM, Thompson MM, Holt PJ. Indications for catheter-directed thrombolysis in the management of acute proximal deep venous thrombosis. Arterioscler Thromb Vasc Biol. 2010;30(4):669-74.

63. Hilleman DE, Razavi MK. Clinical and economic evaluation of the Trellis-8 infusion catheter for deep vein thrombosis. J Vasc Interv Radiol. 2008;19(3):377-83.

64. Kasirajan K, Gray B, Ouriel K. Percutaneous AngioJet thrombectomy in the management of extensive deep venous thrombosis. J Vasc Interv Radiol. 2001:12(2):179-85.

65. Parikh S, Motarjeme A, McNamara T, Raabe R, Hagspiel K, Benenati $\mathrm{JF}_{\text {, et }}$ al. Ultrasound-accelerated thrombolysis for the treatment of deep vein thrombosis: initial clinical experience. J Vasc Interv Radiol. 2008;19(4):521-8.

66. Liu F, Lü P, Jin B. Catheter-directed thrombolysis for acute iliofemoral deep venous thrombosis. Ann Vasc Surg. 2011;25(5):707-15 